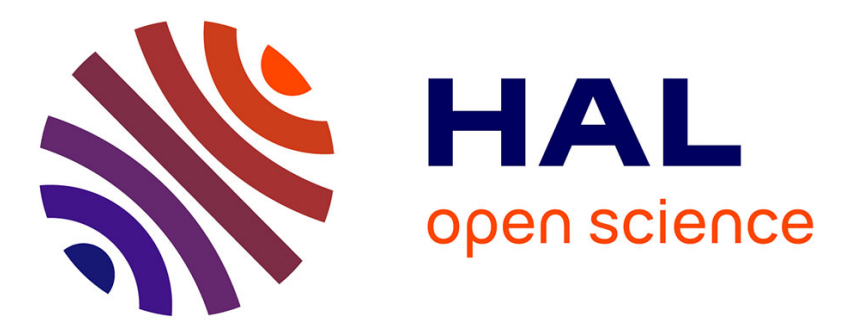

\title{
FÉMINISMES : THÉORIE ET POLITIQUE
}

Ute Gerhard, Jane Jenson, Anne-Marie Devreux, Jacqueline Heinen, Roland Pfefferkorn

\section{To cite this version:}

Ute Gerhard, Jane Jenson, Anne-Marie Devreux, Jacqueline Heinen, Roland Pfefferkorn. FÉMINISMES : THÉORIE ET POLITIQUE : Réflexions à partir des cas allemand, canadien et québécois. Cahiers du Genre, 2006, 1, pp.159-179. hal-01291999

\section{HAL Id: hal-01291999 https://hal.science/hal-01291999}

Submitted on 22 Mar 2016

HAL is a multi-disciplinary open access archive for the deposit and dissemination of scientific research documents, whether they are published or not. The documents may come from teaching and research institutions in France or abroad, or from public or private research centers.
L'archive ouverte pluridisciplinaire HAL, est destinée au dépôt et à la diffusion de documents scientifiques de niveau recherche, publiés ou non, émanant des établissements d'enseignement et de recherche français ou étrangers, des laboratoires publics ou privés. 


\title{
FÉMINISMES : THÉORIE ET POLITIQUE \\ Réflexions à partir des cas allemand, canadien et québécois \\ Ute Gerhard et al.
}

\section{L'Harmattan | Cahiers du Genre}

\author{
2006/3 - HS n ${ }^{\circ} 1$ \\ pages 159 à 179
}

\section{ISSN 1298-6046}

Article disponible en ligne à l'adresse:

http://www.cairn.info/revue-cahiers-du-genre-2006-3-page-159.htm

Pour citer cet article :

Gerhard Uteet al., « Féminismes : théorie et politique » Réflexions à partir des cas allemand, canadien et québécois, Cahiers du Genre, 2006/3 HS n¹, p. 159-179.

Distribution électronique Cairn.info pour L'Harmattan.

(c) L'Harmattan. Tous droits réservés pour tous pays.

La reproduction ou représentation de cet article, notamment par photocopie, n'est autorisée que dans les limites des conditions générales d'utilisation du site ou, le cas échéant, des conditions générales de la licence souscrite par votre établissement. Toute autre reproduction ou représentation, en tout ou partie, sous quelque forme et de quelque manière que ce soit, est interdite sauf accord préalable et écrit de l'éditeur, en dehors des cas prévus par la législation en vigueur en France. II est précisé que son stockage dans une base de données est également interdit. 


\title{
Féminismes : théorie et politique
}

\author{
Réflexions à partir des cas \\ allemand, canadien et québécois
}

\section{Ute Gerhard et Jane Jenson}

Interview par Anne-Marie Devreux et Jacqueline Heinen

Nous nous adressons à vous deux en tant que témoins historiques du développement du mouvement féministe dans vos pays respectifs, des témoins ayant un point de vue singulier. Toutefois, plutôt que d'adopter une trame "historique ", nous avons préféré partir des acquis et des concepts liés au féminisme " consolidé » en sciences sociales et vous interroger sur leur impact sur la société et sur les discours qui y sont prégnants. Comment les théorisations et résultats des recherches féministes ont-ils imprégné les représentations sociales communes et les catégorisations scientifiques, c'est-à-dire les représentations savantes ? En particulier, comment ont-ils permis des corrections d'oublis ou d'erreurs?

Autrement dit, en quoi les acquis du féminisme et les concepts qu'il a contribué à faire émerger en sciences sociales ont-ils influencé des changements dans la société, des changements dans les discours, mais aussi l'évolution du féminisme lui-même?

- Pour commencer, si vous deviez illustrer l'influence des théorisations féministes sur les représentations sociales " ordinaires » 
et sur les représentations savantes des problèmes sociaux touchant les femmes, quel exemple mettriez-vous d'abord en avant?

Ute Gerhard. Avant de tenter de répondre à cette question, je voudrais faire une observation préalable, qui me paraît très importante pour la compréhension de ce qui se joue en Allemagne. Je ne m'exprime pas seulement en tant que personne insérée dans un contexte géographique et politique spécifique, je le fais aussi à un moment donné, marqué par un processus accéléré de transformations sociales, dont on ne saurait sous-estimer le poids. La période qui s'est ouverte en 1989 avec le tournant politique ayant conduit à la réunification des deux États allemands constitue une césure historique, notamment pour le féminisme et les politiques en direction des femmes, qui a profondément modifié les discours et les priorités politiques. On est confronté à une situation grosse de contradictions, de discontinuités et de paradoxes : d'un côté, on note un recul dans la prise en compte des questions de genre, de l'autre, on relève des progrès significatifs, depuis le début des années 1990, tant du point de vue institutionnel que dans le domaine de l'enseignement supérieur, de la formation et de la recherche. Il est difficile d'apprécier la portée des phénomènes antinomiques qui sont à l'œuvre, et le jugement formulé dépend autant des critères retenus que des attentes quant à ce que l'on estime réaliste ou souhaitable. C'est pourquoi je tiens à souligner d'emblée que je fais partie d'une génération qui, avec le mouvement des femmes des années 1970, aspirait à changer radicalement le monde et tout au moins les rapports sociaux de sexe. Et je ne peux pas plus faire abstraction, dans mon jugement politique, des objectifs que nous n'avons pas atteints que des retours en arrière ou des barrières mentales et structurelles faisant obstacle à l'égalité des sexes. Je constate que le mouvement social et politique des femmes est à l'évidence dans une impasse, et j'en déduis que nous avons encore un long chemin à parcourir. Toutefois, en m'appuyant sur les connaissances acquises en sciences sociales, je m’efforcerai d'analyser quelles sont les raisons d'un tel état de fait, ce qu'on peut dire de la situation actuelle et aussi quels changements ou progrès ont été accomplis.

La discussion sur l'égalité des sexes paraît obsolète dans la mesure où, tant dans les représentations collectives que dans le 
discours politique, l'égalité en droit des hommes et des femmes est posée comme nécessaire et - là réside le problème présentée comme allant de soi. Il s'agit d'un point de vue " politiquement correct ", affiché par tous les programmes électoraux, et qui passe plus généralement comme un signe de reconnaissance entre ceux qui défendent des positions progressistes, dans le processus de modernisation en cours. C'est la raison pour laquelle les chercheuses féministes, conscientes de la persistance et de la résistance des multiples inégalités qui touchent les femmes, ne voient là qu'une rhétorique de l'égalité (Wetterer 2003), pour ne pas dire une rhétorique tout court, mise au goût du jour. En effet, le savoir produit par les théories féministes quant aux différences de sexe et aux changements intervenus dans les rapports de genre est largement sous-tendu par l'inégalité même de ces rapports et par des pratiques différentes, selon que l'on est homme ou femme. Le postulat que les uns et les autres sont égaux et qu'il s'agit là d'une chose naturelle bute sur l'expérience concrète des différences qui continuent à imprégner les structures hiérarchiques et les institutions. Néanmoins, ces fractures et ces contradictions sont masquées, voire niées par des théories sociologiques sur l'individualisation et par un bon sens néolibéral selon lequel chacun et chacune, en vertu de la dissolution de liens anciens et de nouvelles libertés de choix, serait responsable de son propre destin et forgerait son propre bonheur.

On en veut pour preuve le maintien de la division sexuelle du travail, tant dans la sphère privée, au quotidien, que dans la ségrégation du marché de l'emploi. C'est pourtant dans le domaine du travail que le mouvement des femmes et la recherche féministe ont le plus marqué les esprits et révolutionné les idées. La critique de la division traditionnelle du travail, en particulier quant à la valeur des tâches domestiques et éducatives et quant à leur invisibilité a été le levier de campagnes politiques et le moteur de mobilisations sociales de femmes allant bien au-delà du milieu académique. Le concept de travail, renouvelé et élargi de façon à englober toutes les activités de prise en charge ou de soins aux autres (le care), constitue à ce jour la pierre angulaire des analyses et des politiques sociales féministes. Toutefois, les succès sont des plus limités : les capacités de résistance et les 
obstacles au changement l'emportent dans les structures comme dans les pratiques traditionnelles. Malgré des progrès sensibles sur le plan éducatif et en dépit de meilleurs résultats ainsi que de l'élévation du niveau de formation des jeunes femmes, le marché de l'emploi reste structuré selon le sexe : $70 \%$ des cursus professionnels relèvent de secteurs typiquement sexués, où les femmes continuent à gagner moins et à bénéficier de moindres chances d'avancement. En outre, le pourcentage de femmes diminue aussi de façon inversement proportionnelle avec le degré de responsabilité. En Allemagne, cela se traduit notamment par le fait que $94 \%$ des postes les plus prestigieux de l'enseignement supérieur — les fameuses chaires universitaires — sont occupés par des hommes. Bien que les jeunes adultes des deux sexes soient plus « égaux » que jamais, les femmes continuent à prendre en charge l'essentiel des tâches familiales et domestiques dès qu'il y a des enfants, et tendent de ce fait à mettre en marge leurs ambitions professionnelles, au moins pour un temps. C'est ainsi qu'en Allemagne de l'Ouest, les congés parentaux sont pris par les femmes dans $95 \%$ des cas et que la plupart des mères ne reprennent le travail qu'à temps partiel ensuite. Dans cette partie du pays, un tiers seulement des mères actives professionnellement travaillent à temps plein. En revanche, en Allemagne de l'Est où les femmes continuent à adhérer au modèle du travail à temps plein, cette proportion atteint encore $70 \%$. Pourtant, là aussi elles gagnent moins que leurs compagnons, et là aussi elles assument l'essentiel du travail au sein du foyer.

Compte tenu de ces inflexions dans les trajectoires féminines et en dépit de situations de départ identiques à celles des jeunes hommes, les biographies professionnelles et familiales des jeunes femmes tendent à ressembler à celles de leurs mères quant aux implications qui en découlent : dépendance personnelle, revenus inférieurs et surtout pauvreté bien plus grande chez les femmes âgées. Le fait est que cette différence des sexes, à la fois nouvelle et ancienne, est très peu prise en compte, notamment dans une optique politique, car la critique porte peu sur les rapports sociaux à l'œuvre, qu'il s'agisse des normes masculines qui prévalent sur le marché de l'emploi, des carences de l'infrastructure concernant la petite enfance ou du manque de soutien concret de la part de l'entourage, à commencer par le partenaire. 
Visiblement, les arrangements respectifs au sein du couple dépendent surtout de décisions individuelles. L'aspiration à l'égalité et le désir de se réaliser passent pour acquis dans les couples modernes et sont hautement valorisés dans les médias, mais l'une et l'autre continuent à se heurter à l'image traditionnelle de la « bonne mère ».

Jane Jenson. Tout comme Ute Gerhard, je pense qu'il est utile de faire quelques remarques préliminaires avant de répondre à vos questions sur le féminisme canadien et québécois. La première est qu'on n'a pas affaire à un seul mouvement de femmes ou à un seul féminisme dans le pays. Depuis les années 1960, il existe un mouvement de femmes au Québec dont la formation a été marquée avant tout par la principale question en jeu dans la province, à savoir celle de l'indépendance et du rapport entre le Québec et le Canada. Dans le reste du pays, le mouvement des femmes a lui aussi été profondément marqué par le mouvement nationaliste, mais il s'agissait du mouvement nationaliste canadien et du rapport entre le Canada et les États-Unis. Les deux pans du mouvement des femmes " canadien » ont travaillé de concert des années 1960 jusque dans les années 1980, mais depuis la fin des années 1980, ils ont pris des directions complètement différentes.

La seconde remarque est qu'une bonne partie du travail académique sur le féminisme a consisté à analyser la relation entre un mouvement et l'État. Certaines féministes ont toujours été méfiantes à l'égard de l'État, mais je crois pouvoir affirmer sans trop de controverse que, tant au Québec qu'au Canada, les féministes se sont montrées désireuses de travailler avec l'État et au sein de celui-ci et qu'elles ont consacré beaucoup d'attention aux politiques publiques ainsi qu'aux politiques électorales.

Une troisième remarque liminaire est que, à la fois au Québec et au Canada et tout comme en Allemagne, l'influence du mouvement des femmes a profondément diminué, pour ne pas dire que ce dernier est moribond. À l'extérieur du Québec, le déclin a été brutal dans les années 1990, alors que le mouvement était isolé et très critiqué et que l'État a mis fin au soutien qu'il avait apporté jusque-là aux institutions du « féminisme d’État ». Depuis lors, il a été virtuellement réduit au silence et la situation des 
femmes en général ne fait plus l'objet de débats publics, d'actions ou d'attention politiques. Au Québec, le déclin est intervenu plus tard. Mais là aussi, la visibilité du mouvement s'est fortement réduite et l'État québécois procède actuellement de son côté au démantèlement des institutions du féminisme d'État.

Or il faut souligner que tout cela s'est passé en dépit de la présence d'un nombre significatif de femmes — quand bien même il est insuffisant - dans des postes de responsabilité politique, tant dans les instances élues que dans les administrations publiques, dans les médias et à tous les niveaux de l'économie.

Si l'on examine la production académique, notamment en science politique et en sociologie, on voit qu'elle a été fortement marquée par cette histoire. Les analyses concernant la participation des femmes et leur représentativité ont nourri la tradition consistant à identifier le nombre de femmes, la place des femmes, etc., dans chaque instance politique ainsi que dans les administrations publiques. Les recherches ont par ailleurs soutenu les pratiques des associations ainsi que celles de l'État en termes d'ouverture d'espaces politiques pour les femmes : sièges garantis aux tables de concertation régionale au Québec, par exemple, ou dans les partis politiques et syndicats.

Au cours des dernières années, l'intérêt pour la participation active des femmes à la politique a ouvert un certain nombre de voies de recherche. À part les études électorales traditionnelles, une voie de recherche, plus innovante celle-là, se situe directement dans la lignée des analyses des mouvements de femmes. C'est le cas de l'examen détaillé de la mobilisation des femmes à l'intérieur de leurs organisations, de même que dans les organisations mixtes. Dominique Masson (2001, p. 91), par exemple, a étudié la participation des femmes dans les institutions régionales québécoises. Elle écrit que "l'arrivée des groupes de femmes parmi les acteurs avec qui se partage la gouverne du développement régional a permis [...] d'élargir le système de représentation des Conseils régionaux de développement (CRD) à l'identité-femmes, aux intérêts qui lui sont associés et aux actrices qui en sont les porteuses ».

Une dernière voie de recherche concerne la participation des femmes et des féministes dans les mouvements nationalistes 
canadien et québécois. Les féministes canadiennes ont payé un prix énorme pour leur opposition aux traités de libre échange avec les États-Unis et le Mexique. Le mouvement des femmes québécois, comme nous l'avons dit plus haut, a grandi dans le sillage de la Révolution tranquille. La relation avec le mouvement nationaliste, suivie de très près par Diane Lamoureux entre autres (2001), a été aussi compliquée que celle de tout mouvement de femmes avec ses mouvements frères, qu'il s'agisse du mouvement ouvrier, du mouvement pour la paix ou du mouvement pour les droits civils. Lors du premier référendum, en 1980, la question du soutien des femmes au fédéralisme ou à l'indépendance représentait un enjeu majeur en soi, et en 1995, les enjeux genrés ont été soulevés de nouveau. Le problème est particulièrement épineux pour les femmes francophones hors Québec, dont la survie en tant que minorité culturelle dépend en partie de l'existence d'un Canada prônant le bilinguisme.

Les recherches-action, promues par les organismes publics et soutenues par les associations de la société civile ont eu également des résultats importants. Les sociologues, les politiques et les travailleuses sociales se sont engagés dans un programme de recherche centré sur l'action à l'intérieur des structures et des institutions, qui a lui-même donné lieu à un corpus d'études importantes sur les stratégies poursuivies par les associations de femmes et leurs alliés, de même que sur les intérêts de l'État et les conséquences de ses choix politiques pour l'autonomie des femmes.

Les conséquences du néolibéralisme pour le genre représentent un sujet de préoccupation majeure des féministes. Les études, souvent vulgarisées dans les médias et auprès des groupesfemmes, observent que le néolibéralisme a entraîné : une perte de légitimité des groupes mobilisés pour formuler des revendications au nom des femmes ; une perte de bons emplois pour les employées de l'État, des coupures ayant été faites dans les dépenses publiques ; enfin, une réduction de prestations particulièrement importantes pour les femmes. En d'autres termes, les droits de citoyenneté des femmes ont été réduits, à la fois dans le domaine politique et dans le domaine social. On observe également que la transformation du régime de citoyenneté canadien dans la deuxième moitié des années 1990, s’est opérée 
en mettant davantage l'accent sur les enfants que sur les besoins des femmes ; ce qui a eu des conséquences sur la possibilité pour les femmes d'accéder à une citoyenneté à part entière.

- Quel est l'impact des théories et des résultats des recherches féministes sur les choix politiques et sur les catégories soustendant ces choix (démocratie, égalité, droit...)?

Ute Gerhard. Tant le mouvement des femmes que les recherches féministes qui l'ont accompagné ont entraîné des transformations durables en matière de conscience individuelle et dans les rapports sociaux de sexe, qu'il s'agisse du discours sur la sexualité, du droit à disposer de son propre corps, de la liberté reproductive des femmes ou du scandale que constituent les violences contre les femmes. Il en est découlé un processus collectif d'apprentissage qui a eu des répercussions à l'échelle mondiale grâce aux conférences mondiales des femmes de l'ONU et qui a contribué à ce que le débat sur les droits des femmes soit posé en termes de droits humains. Les connaissances relatives au point nodal des rapports sociaux de sexe, tout comme la liberté beaucoup plus grande (et ressentie comme naturelle) dans les relations sexuelles n'ont pas peu contribué à l'expression d'un nouveau pluralisme dans les formes d'existence et passent pour une révolution culturelle des rapports de genre. On en veut pour preuve toutes les conquêtes légales, qui paraissaient impensables voici trente ans : depuis l'égalité des enfants nés hors mariage jusqu’à la décriminalisation de l'homosexualité, en passant par la réforme du divorce et la proclamation du principe d'égalité dans le droit du mariage, sans oublier la reconnaissance officielle de la vie commune des couples homosexuels (le mariage "gay »), la pénalisation des violences conjugales, l'amélioration de la protection des femmes et des jeunes filles contre les violences, etc. En Allemagne, seule la revendication centrale du mouvement des femmes des années 1970 — à savoir le droit de décider librement d'interrompre une grossesse continue à être soumise à une réglementation très stricte et à dépendre de l'approbation des médecins. Les querelles très violentes à ce propos, auxquelles les Églises n'ont cessé de se mêler, font de cette question un ultime bastion symbolique du pouvoir patriarcal. 
Que les profondes transformations dans la compréhension des rapports sociaux de sexe et dans les représentations quant aux rôles assignés se heurtent aux inégalités persistantes dans les conditions matérielles évoquées plus haut témoigne de la totale incohérence de la politique d'égalité des sexes et désigne les points de rupture en la matière. Ces problèmes se traduisent actuellement par le caractère explosif du débat politique concernant le recul des naissances qui, conjointement au vieillissement de la population, tend à bouleverser l'équilibre démographique et mettrait ainsi la sécurité sociale en danger, en raison de la rupture du contrat de solidarité entre les générations. Il est de fait que, face à l'impossibilité de combiner famille et travail professionnel, la réponse de nombreuses femmes ayant un bon niveau de formation est de ne pas faire d'enfants ou de reculer l'âge à la première naissance. La question se pose donc de savoir si le débat sur l'avenir de notre société pourra servir de levier politique en faveur des femmes. Néanmoins, face à la complexité des problèmes posés, qui ont en outre une dimension transnationale, les acteurs politiques susceptibles de mettre en œuvre une politique égalitaire dans le domaine des rapports sociaux de sexe font visiblement défaut.

- Les changements observés en politique ont-ils un lien avec le rôle du féminisme d'État dans votre pays ou sont-ils à imputer avant tout à la force des groupes (du mouvement) féministes ?

Jane Jenson. Les analyses féministes canadiennes et québécoises ont évolué en rapport étroit avec les institutions et les chercheurs et chercheuses travaillant à l'intérieur de l'État.

Au Québec, l'appréciation des inégalités de genre et de l'existence de discriminations structurées et systémiques a été nourrie par deux sources principales. D'abord, la mobilisation du mouvement des femmes. À partir du milieu des années 1960, ce mouvement a rapidement engendré une analyse politique, sociologique et historique, au sein de groupes clés comme les groupes féministes autonomes, mais aussi au sein des syndicats et des partis politiques, ou dans les universités. Le second facteur auquel on doit l'essor de cette tradition d'analyse est l'acceptation précoce par des institutions intellectuelles clés (universités, organismes financeurs et organismes de recherche gouverne- 
mentaux) de la légitimité non seulement des analyses de la condition féminine, mais aussi d'analyses politiques féministes.

La Révolution tranquille, moteur de la modernisation du Québec, a eu pour conséquence significative une puissante seconde vague féministe. Marie Lavigne, présidente du Conseil du statut de la femme du Québec, a décrit les années 1960 ainsi :

On assiste aussi à l'époque [de la Révolution tranquille] à une renaissance du mouvement des femmes, qui s'alimente aux grands courants du féminisme contemporain. Toute une kyrielle de nouveaux groupes de femmes s'ajoutent alors aux grandes associations féminines existantes. Ces années voient également l'émergence d'une action militante des femmes à l'intérieur des structures syndicales et politiques (Andrews, Rodgers 1997).

Le Canada a suivi de très près.

Cette mobilisation a eu pour effet immédiat, entre autres, que la Fédération des femmes du Québec (FFQ, créée en 1966) a exercé une pression sur le gouvernement du Canada, avec l'appui d'associations et de groupes canadiens anglais, pour obtenir la création d'une commission royale mandatée pour évaluer la condition des femmes et faire des recommandations à ce sujet. Créée en 1967, la Commission royale d'enquête sur la situation de la femme au Canada a rencontré, lors d'une tournée nationale, des groupes du nouveau mouvement de libération de la femme aussi bien que des organisations traditionnelles de femmes. Elle a rendu son rapport en 1970 après un travail de recherche majeur (effectué sous la direction de Monique Bégin) appuyé par des audiences publiques.

D'autres institutions non gouvernementales ont également réagi à la mobilisation du mouvement féministe dans les universités. Les femmes ont réclamé une reconnaissance à l'intérieur de leurs associations disciplinaires : en science politique, à partir des années 1980, les publications évaluant la situation de ce champ font état, en déplorant ces faits, d'une présence limitée des femmes dans le monde universitaire, et d'un manque d'études sur les femmes et le genre.

Ute Gerhard. Le rôle d'un féminisme d’État porté par des actrices que l'on qualifierait de « fémocrates » est étranger au débat allemand, ou du moins il s’agit d'un concept importé. En 
revanche, le fossé séparant le mouvement des femmes dit "autonome ", à savoir indépendant de tous les partis, et les unions traditionnelles de femmes d'Allemagne de l'Ouest, avec leur politique de lobbying sur le thème de l'égalité, a longtemps été présenté par les deux parties en présence comme un obstacle insurmontable à cause de leurs divergences idéologiques. Et ce, en un temps où les rangs du mouvement des femmes grossissaient grâce aux sympathies suscitées dans de nouveaux regroupements politiques tels que le mouvement de la paix, les syndicats, les Églises, ou même dans les partis traditionnels. Le parti des Verts jeta un premier pont au début des années 1980 en instaurant de nouveaux critères et en engageant une politique égalitaire en matière de représentation - une direction exclusivement féminine fut même élue au départ à la tête du parti (on parla de «Feminat »). Parallèlement, les mesures d'égalité des chances prises au niveau fédéral dans un premier temps — celui des Länder - et au niveau communal dans un second temps, instaurèrent un nouveau champ d'intervention politique avec la création de comités de l'égalité des chances, de Bureaux de femmes et de déléguées féminines institutionnalisant tout un système administrativo-politique. À l'heure qu'il est, il existe en Allemagne quelque 1900 Bureaux de l'égalité des chances au sein des communes, des associations ou des institutions. Les femmes qui se sont vu confier ces charges soit étaient des féministes de toujours, soit le sont devenues grâce à cette activité.

Enfin, le mouvement autonome des femmes a été à l'origine de l'institutionnalisation dans les universités, sous des formes multiples, des recherches sur les femmes - aujourd'hui, des études de genre. Il a permis d'obtenir entre-temps une centaine de chaires de professeur spécialement consacrées à ces recherches, des filières universitaires spécifiques ont pu être développées, y compris des programmes de promotion et des formations diplômantes, sans compter les centres de recherche consacrés aux études féminines. Malgré ces réussites, on peut cependant s’interroger sur les limites de ces recherches désormais intégrées dans les universités. Dans quelle mesure parviennent-elles à conserver leur caractère critique et à faire prendre en compte la problématique du genre dans les courants dominants des diverses disciplines scientifiques? 
En effet, au cours des années 1970, les actrices concernées avaient tissé des liens étroits entre le mouvement des femmes, la recherche féminine, l'action politique en faveur des femmes et le féminisme. Une telle interdépendance ne va plus de soi : aujourd'hui, on peut faire des recherches sur les femmes ou le genre sans avoir une conscience féministe, voire sans s'intéresser à la politique mise en œuvre en faveur des femmes. D'autre part, aujourd'hui, des jeunes femmes peuvent se dire féministes sans avoir participé à un mouvement de femmes. Leur prise de conscience est avant tout intellectuelle, elle s'est faite au travers de lectures et de l'étude des structures inégalitaires qui, hier comme aujourd'hui, continuent à déterminer leur propre existence. La professionnalisation et l'institutionnalisation de la recherche sur les femmes ou le genre ont en outre conduit à une division du travail et à un approfondissement des différenciations dans les divers champs de la recherche et de l'action politique. Mais elles ont aussi un coût lorsqu'elles se traduisent non seulement par une distanciation hautaine vis-à-vis du qualificatif " féministe », mais également par une nette tendance à freiner le mouvement critique en direction du changement et des conditions sociales.

- Les recherches féministes ont-elles conduit à l'ouverture de nouveaux «dossiers », à la "censure » de certains thèmes, à des vulgarisations déformantes de résultats ? Quels sont les thèmes ou les champs absents, ou trop peu travaillés, par les chercheuses féministes de votre pays ?

Ute Gerhard. J'ai déjà évoqué les nouvelles perspectives et les nouveaux champs d'action ouverts par la recherche féministe (l'extension du concept de travail ; la libéralisation de la sexualité ; la problématisation de la violence dans les relations entre les sexes ; la modification des modes de vie, la révolution culturelle dans les représentations de la masculinité et de la féminité). Par contre, à mon avis, les demandes et les besoins fondamentaux de la majorité des femmes sont négligés, voire déformés, dans les discussions très sophistiquées autour des théories féministes, notamment autour de la déconstruction de la catégorie de genre, dans lesquelles s'engagent avec un certain brio les jeunes chercheuses très au fait sur le plan théorique. La distance entre la théorisation féministe et les « laborieuses 
avancées sur le plan politique » devient abyssale lorsque des chercheuses ne parviennent plus à traduire leurs savoirs théoriques dans la langue de l'action politique ou de la praxis quotidienne, voire si cette médiation ne les intéresse même pas. Cet intellectualisme - d'aucuns parlent du snobisme de la théorie féministe - peut constituer une forme de distanciation, à la fois vis-à-vis des objectifs que ces chercheuses considèrent comme dépassés, et vis-à-vis de l'engagement des féministes plus âgées. De part et d'autre, cette attitude conduit à la déstabilisation et à l'absence de communication.

Jane Jenson. Après la commission royale et dans les années 1970 et 1980, les institutions ainsi que les féministes à l'extérieur ont repris les questions d'égalité politique et économique déjà soulevées par la commission royale ; elles ont également rapidement étendu le champ de la recherche, analysant en détail, par exemple, les questions de la violence conjugale et des nouvelles techniques de reproduction, domaines que la commission royale avait presque totalement laissés de côté.

Bien sûr, dans les années 1970 et 1980, de nombreuses féministes radicales ne faisaient pas confiance à l'État. Elles craignaient que les projets féministes (par exemple dans les domaines des soins de santé et de la violence conjugale) qui avaient grandi de manière autonome, ne soient récupérés par l'État et ne deviennent moins radicaux s'ils devaient être financés par des fonds publics. Cependant, dans une évaluation de l'expérience publiée vers la fin des années 1990, Diane Lamoureux conclut que paradoxalement, le rôle réduit joué par l'État néolibéral et sa réticence à s'engager dans des services nouveaux et chers ont permis aux féministes de conserver la maîtrise de leurs programmes. Cependant, elles ont dû "répartir entre elles la pauvreté » (Andrew, Rodgers 1997).

Les préoccupations ont récemment pris un tour nouveau. D’abord, on examine maintenant de manière plus détaillée le rapport entre multiculturalisme et féminisme, dans le contexte de politiques canadiennes qui reconnaissent la diversité culturelle. Ensuite, la montée du nationalisme chez les peuples autochtones du Canada a suscité un certain nombre d'analyses sur les manières dont l'égalité entre les genres et le nationalisme autochtone 
pouvaient entrer en conflit, de même que sur les spécificités des revendications des femmes autochtones à l'intérieur du mouvement des femmes en général.

Un nouveau dossier peut être nommé « le mondial » ou « l'international ». Jusqu'à récemment, l'accent était fortement mis sur les situations canadienne et québécoise. Cet intérêt pour la proximité s'explique en grande partie par les origines de la recherche elle-même. L'objet de la recherche étant intimement lié aux mouvements de femmes québécois et canadien dont il tirait sa force, il n'est pas surprenant qu'il soit demeuré le même et reste axé sur les comportements, les pratiques, les discours et les conflits dans ces mouvements, dans toute leur complexité.

Depuis quelques temps cependant, un nouveau type de recherche émerge, qui reflète à la fois les préoccupations actuelles du féminisme et le fait que la mondialisation occupe une position centrale en politique ici et ailleurs. L'analyse des femmes et du genre se tourne vers l'extérieur. Deux exemples illustrent ce changement.

En 2002, Chantal Maillé présente un numéro de Recherches féministes (2002) intitulé « Migrations : femmes, mouvement et "refondation" du féminisme ». On y relève une double optique. Certains des six articles proposés analysent des phénomènes transnationaux, comme le trafic des femmes. D'autres, cependant, restent consacrés au Québec, mais élaborent leur analyse du point de vue de femmes marginalisées à l'intérieur de cette société, en particulier les immigrantes récentes. La problématique du numéro met au défi les féministes québécoises de reconnaître leur propre utilisation d'un "métarécit d'une société québécoise blanche, homogène, de métissage récent, où sont venus des gens d'ailleurs dans le dernier quart de siècle ».

D’un point de vue historique, ce métarécit est, bien sûr, inexact. La société canadienne, comme toutes celles d'Amérique du Nord, a toujours été une société d’immigration, qui a absorbé un très grand nombre de nouveaux venus depuis le XVII ${ }^{\mathrm{e}}$ siècle, d'abord d'Europe, et récemment, d'autres continents. Le premier métissage s'est fait avec la rencontre des peuples autochtones et il s'est poursuivi alors que la société absorbait des vagues d’immigration européenne. Cependant, la conscience de ce plu- 
ralisme atteint actuellement un niveau sans précédent. Le mouvement nationaliste québécois cherche à se définir en termes de citoyenneté plurielle plutôt que s'inscrire dans une culture nationale unique et le mouvement nationaliste canadien affiche sa « diversité » dans une société multiculturelle. Les féministes ont été touchées par ce processus d'élargissement sociétal, d'où le thème de la « refondation » du féminisme.

Les recherches sur le féminisme après la Marche mondiale des femmes de l'an 2000 fournissent une deuxième nouvelle direction. Elle reflète une conscience de l'importance du transnational et du mondial et est particulièrement attrayante pour la jeune génération.

La marche représentait une première expérience de mobilisation des mouvements féministes de plus de cent cinquante pays du monde avec des revendications proprement sociales pour lutter contre la pauvreté et la violence, adressées aux États nationaux comme aux instances internationales. Elle était une initiative de la Fédération des femmes du Québec, suite à sa propre marche nationale, " Du pain et des roses », en 1995. Mobilisation mondiale, la marche a également demandé aux féministes un travail de collaboration pour faire émerger un consensus minimum, en particulier avec des femmes engagées dans une démarche religieuse.

Que cette initiative transnationale particulière atteigne ou non ses objectifs, il est important de noter que de telles recherches constituent une nouvelle génération d'analyse féministe, génération qui regarde au-delà des frontières nationales non seulement parce que la comparaison présente un intérêt, mais aussi parce que la réalité de notre monde force la jeune génération à regarder vers l'extérieur.

- Comment la question des «races» et celle des «classes» ont-elles été prises en compte par la pensée et la recherche féministes?

Ute Gerhard. Pendant longtemps en Allemagne le concept de « race », ou plutôt la discussion autour du racisme, n’a été problématisée que par rapport au débat critique portant sur les crimes nazis. C'est aussi la raison pour laquelle ce concept a été en partie refoulé ou plutôt repoussé vers le passé. Ce n’est que 
progressivement qu'on s'est aperçu que la République fédérale allemande était un pays d'immigration avec tous les problèmes qui en découlent - problèmes que le concept de société multiculturelle ne permet pas de penser de façon simple. Le concept de " race » a néanmoins été écarté en raison de ses connotations politiques et, comme dans la recherche anglo-saxonne, il est remplacé par celui d'ethnicité. On présume que ce dernier renvoie moins à la dimension biologique qu'à l'identification de l'origine socioculturelle et géographique. De nombreuses recherches en histoire des femmes ou en histoire du genre ont vu une génération de petites-filles de plus en plus impartiales assumer sans ménagement la confrontation avec le nazisme, notamment à propos de la complicité de femmes ou de l'antisémitisme des organisations du premier mouvement de femmes. À côté de ces travaux historiques, à partir du début des années 1990, les chercheuses féministes ont aussi participé en République fédérale à la critique postcoloniale et poststructuraliste qui avait alors cours dans le monde entier. Les femmes les plus jeunes, surtout, ont participé avec un grand empressement au cultural turn (tournant postmoderne) dans la production théorique féministe.

L'examen des conditions de vie les plus diverses, y compris parmi les femmes, et la remise en question théorique et politique d'un " nous » féministe a conduit à des débats extrêmement véhéments, tout particulièrement entre les différentes classes d’âge ou "générations ", et, comme quelques observatrices le pensent, à une "crise des fondements » du féminisme en tant que théorie politique. Jusqu'à quel point la déconstruction du sujet collectif "femmes » a-t-elle entraîné la fin, voire l'impossibilité d'un mouvement commun des femmes ? Les avis à ce propos sont aujourd'hui très partagés.

Entre-temps, la fumée s'est un peu dissipée au-dessus de la mêlée. Les théoriciennes féministes parlent désormais d'une confrontation productive, assortie de contradictions et de paradoxes, dans la mesure où l'approche féministe critique concernant les rapports sociaux de sexe et les différences entre femmes offre un potentiel réflexif pouvant s’appliquer aux problématiques les plus diverses. Confrontées à celle de la marginalisation, qui a contribué à les éclairer, les féministes ont plus particulièrement appris à mesurer l'importance des divers « axes de la 
différence » (Knapp, Wetterer 2003). Dans l'ardeur de la lutte et dans l'énumération de plus en plus ritualisée des différences structurelles et culturelles les plus visibles (race, classe, ethnicité, sexe, âge, orientation sexuelle, conviction religieuse ou politique), généralement suivie d'un " etc. », on a effectivement oublié à quel point - notamment dans les années 1970, et plus précisément parmi les féministes qui prenaient leurs distances vis-à-vis des partis de gauche - les débats furent parfois intenses et à quel point il fut alors nécessaire de changer de perspective en mettant l'accent sur les rapports sociaux de sexe, dans un contexte où les théories sociologiques sur l'appartenance de classe et sur les inégalités sociales étaient hégémoniques.

À l'opposé, les théories «de l'articulation » restent aujourd’hui très indéterminées quant au poids respectif accordé à ces « axes »: dans les recherches sur l'inégalité, y compris dans les recherches féministes, la superposition de ces multiples différences est plutôt abordée dans le cadre d'une division par champs (par exemple dans la recherche sur les migrations, dans les études de sociologie des religions consacrées aux musulmanes en Allemagne) sans pour autant rendre superflue ou inutile la distinction entre sexes comme structure centrale dans toutes les sociétés. Ce qui fait l'originalité de la recherche féministe et qui, en même temps, la rend suspecte aujourd'hui comme hier, ce sont ses débats publics passionnés, dans lesquels il s’agit toujours de clarifier des positions tout en s'interrogeant sur les conceptions de la justice.

Jane Jenson. Le concept de classe a été mobilisé par de nombreuses féministes dans les années 1960. Les féministes socialistes et marxistes appliquaient ce concept aux critiques du patriarcat ainsi qu'aux relations sociales entre les femmes. Son usage s'est réduit au cours des dernières années, parallèlement au déclin des analyses matérialistes et structuralistes. Le concept de race a lui aussi été utilisé dans un très grand nombre d'études, en particulier en anglais. Il est généralement intégré dans le trio " classe-race-genre " visant à désigner les principaux ensembles sociétaux qui structurent la vie des femmes. La façon dont les femmes "racisées " sont confinées dans les emplois les plus dévalorisés et souvent employées par d'autres femmes dans des travaux domestiques a notamment fait l'objet 
d'une très grande attention. Au Canada, les féministes considèrent comme allant de soi la nécessité de distinguer les femmes entre elles en fonction de leurs différences et de leurs identités - que ces distinctions aient trait à la classe, la race, l'ethnie ou autres.

- Comment la relation entre division du travail et construction du genre ou rapports sociaux de sexe est-elle actuellement conçue?

Ute Gerhard. J'ai déjà souligné, dans ma réponse à la première question, le caractère central dans la recherche et l'action politique féministes du concept de travail ainsi que de l'organisation sociale des différents travaux socialement nécessaires (le travail professionnel comme le travail domestique et d'éducation). Je considère qu'une organisation du travail équitable du point de vue des sexes sociaux serait le levier décisif pour que le processus de modernisation des rapports de genre - qui est avant tout d'ordre culturel — passe du niveau des discours à celui de la pratique et du changement des structures sociales.

- L'antiféminisme a-t-il évolué dans votre pays ? Quelles sont, selon vous, ses formes actuelles? Dans quel domaine se fait-il le plus sentir?

Jane Jenson. Actuellement, les mouvements de femmes canadien et québécois font face à une exclusion quasi totale de la place publique : il sont invisibles, méprisés des médias, etc.

Dans la deuxième moitié des années 1990, le gouvernement du Canada a éliminé toutes les instances étatiques qui représentaient "les femmes » (créées dans le sillage du rapport de la commission royale de 1971 — voir plus haut), à part une toute petite unité de recherche. Au Québec, le mouvement des femmes restait présent dans les débats publics jusque dans les années 2000, mais il ne l'est plus actuellement. Puis, en 2005, le gouvernement du Québec a proposé la disparition du Conseil du statut de la femme par sa fusion dans un Conseil « d'égalité ».

À côté, et surtout dans le reste du Canada, les mouvements populistes de droite, les néolibéraux, et les protestants radicaux mettent en question les politiques d'égalité, l’investissement 
public dans les services de garde, et ainsi de suite. Leur vision traditionnelle de la famille l'exige.

Ute Gerhard. Dès le départ, le mouvement des femmes et ses représentantes ont dû s'affronter à l'antiféminisme. Les Françaises ont inventé le terme féminisme vers la fin $\mathrm{XIX}^{\mathrm{e}}$ siècle et l'ont fait connaître dans le monde via les congrès internationaux. Dans la langue allemande, le terme féminisme a d'abord été utilisé par les adversaires pour dénoncer le mouvement des femmes, c'est pourquoi Hedwig Dohm écrivait dès 1902 : Les antiféministes. Un livre en défense des femmes ${ }^{1}$. Depuis le tournant de 1989, l'antiféminisme est redevenu bruyant, populaire et de bon ton dans le débat politique, et pas uniquement en Allemagne. Outre les reculs qui accompagnent toutes les histoires glorieuses, en même temps que les craintes sur la fin annoncée depuis longtemps du mouvement des femmes, se rencontrent ici les camps les plus divers dans une alliance remarquable visant à balayer le féminisme. Cela ne vaut pas uniquement pour les éternels conservateurs - des hommes avant tout qui craignent de perdre leurs privilèges de toujours ; cela concerne aussi des gens soi-disant de gauche pour qui tout cela est allé beaucoup trop loin, politiquement et théoriquement, ou qui dans leurs relations privées n’ont pas supporté l'émancipation de leurs partenaires. Par ailleurs, la rencontre entre les féministes ouest-allemandes et les militantes pour les droits civiques est-allemandes n'a pas conduit au renforcement du mouvement féministe dans l'Allemagne réunifiée, bien au contraire. Les malentendus se sont multipliés et les femmes est-allemandes, qui, dans des conditions difficiles, avaient en principe obtenu depuis longtemps l'égalité des droits, ont pris leurs distances vis-à-vis d'un féminisme occidental perçu comme ennemi des hommes et bien trop acerbe. Enfin, il faut compter en outre avec les filles des militantes féministes, d'un niveau de formation élevé et bien dans leur peau qui, jusqu’à l'entrée dans une profession ou jusqu'à ce qu'elles fondent leur propre famille, n’ont fait l'expérience d'aucun inconvénient lié à leur appartenance sexuelle. De leur

\footnotetext{
${ }^{1}$ «Die Antifeministen. Ein Buch zur Verteidigung ». Hedwig Dohm (18311919) est une figure importante du féminisme allemand de la seconde moitié du $\mathrm{XIX}^{\mathrm{e}}$ siècle, elle a publié de nombreux ouvrages et défendu le droit de vote des femmes dès 1873.
} 
point de vue, le féminisme est dépassé. Elles estiment bien entendu avoir droit à ses conquêtes, mais elles prennent leurs distances vis-à-vis des lamentations de leurs mères et exigent de déterminer elles-mêmes - après tout, leurs aînées se sont-elles battues pour autre chose? - comment elles veulent mener leur vie privée et se mêler des affaires publiques.

Un tel bilan relèverait cependant d'une généralisation inappropriée. Car le concept de la situation de génération, que Karl Mannheim (1928/1969) a développé en référence au rôle des mouvements sociaux dans le changement social, aide à comprendre que les conquêtes, héritage culturel du changement social, ne peuvent jamais être reprises telles quelles par la génération suivante qui, le plus souvent, doit se les réapproprier et les transformer. Certes, beaucoup de ces jeunes femmes disent : " Je ne suis pas féministe », mais elles se sentent indépendantes en pratique et essayent de ne pas se penser d'abord en tant que femmes, mais en tant qu'égales des hommes. Un grand nombre d'entre elles participent à de nouvelles formes de contre-culture, par exemple dans la culture pop, dans le milieu musical. Elles créent leurs propres réseaux, à propos desquels elles partagent leurs expériences, y compris dans le domaine technique, par exemple dans les clubs informatiques, ou s'engagent dans des associations et des initiatives qui relèvent plutôt de la société civile que de la politique institutionnelle. Avec les offres de formation en études de genre, conquises avec peine et toujours à défendre, validées comme unités d'enseignement et conférant une qualification professionnelle, même les universités sont des lieux d'exercice d'une pensée féministe critique ; en lien avec la mobilisation politique, elles permettent une socialisation plutôt intellectuelle et cognitive qu'émotionnelle. Comme les études empiriques comparatives européennes le prouvent (Griffin 2004), l'importance de ces têtes de pont universitaires ne doit pas être sous-estimée et laisse espérer que l'histoire du féminisme, pardelà le flux et le reflux de mouvements cycliques, n’a pas dit son dernier mot.

Traduction de Jacqueline Heinen et Roland Pfefferkorn pour la partie allemande 
FÉMINISME — MOUVEMENT FÉMINISTE — THÉORIE FÉMINISTE — GÉNÉRATIONS — SECONDE VAGUE — INÉGALITES SEXUÉES

\section{Références}

Andrew Caroline, Rodgers Sanda (eds) (1997). Les femmes et l'État canadien = Women and the Canadian State. Montréal \& London, McGill-Queen's University Press.

Griffin Gabriele (ed) (2004). Employment, Equal Opportunities and Women's Studies. Women's Experiences in Seven European Countries. Königstein i.T, Ulrike Helmer Verlag.

Lamoureux Diane (2001). L'amère patrie. Féminisme et nationalisme dans le Québec contemporain. Montréal, Remue-ménage.

Maillé Chantal (2002). Migrations : mouvement et "refondation" du féminisme. Recherches féministes, vol. 15, $\mathrm{n}^{\circ} 2$ " Migrations ».

Masson Dominique (2001). "Gouvernance partagée, associations et démocratie : les femmes dans le développement régional ». Politique et sociétés, vol. 20, $\mathrm{n}^{\circ}$ 2-3 « Gouvernance et société civile ».

Knapp Gudrun-Axeli, Wetterer Angelika (eds) (2003). Achsen der Differenz. Gesellschaftstheorie und feministische Kritik II. Münster, Westfälisches Dampfboot.

Mannheim Karl (1928/1969). Das Problem der Generationen, Reprint von 1928. In Ludwig von Friedeburg (ed). Jugend in der modernen Gesellschaft. Köln/Berlin, Kiepenheuer \& Witsch.

Wetterer Angelika (2003). Rhetorische Modernisierung. Das Verschwinden der Ungleichheit aus dem zeitgenössischen Differenzwissen. In Knapp Gudrun-Axeli, Wetterer Angelika (eds). 